JOURNAL OF SYNCHROTRON RADIATION
Received 18 March 2015

Accepted 18 March 2015

Keywords: synchrotron radiation circular dichroism (SRCD); vacuum-ultraviolet (VUV); secondary structure; protein; denaturation;

CDApps; integrated software

\section{CDApps: integrated software for experimental planning and data processing at beamline B23, Diamond Light Source. Corrigendum}

Rohanah Hussain,* Kristian Benning, Daniel Myatt, Tamas Javorfi, Edoardo Longo, Timothy R. Rudd, Bill Pulford and Giuliano Siligardi*

Diamond Light Source Ltd, Diamond House, Harwell Science and Innovation Campus, Didcot, Oxfordshire OX11 ODE, UK. *Correspondence e-mail: rohanah.hussain@diamond.ac.uk,

giuliano.siligardi@diamond.ac.uk

In the paper by Hussain et al. [(2015), J. Synchrotron Rad. 22, 465-468], Daniel Myatt is missing from the list of authors. The complete list of authors should be Rohanah Hussain, Kristian Benning, Daniel Myatt, Tamas Javorfi, Edoardo Longo, Timothy R. Rudd, Bill Pulford and Giuliano Siligardi.

\section{References}

Hussain, R., Benning, K., Javorfi, T., Longo, E., Rudd, T. R., Pulford, B. \& Siligardi, G. (2015). J. Synchrotron Rad. 22, 465-468.

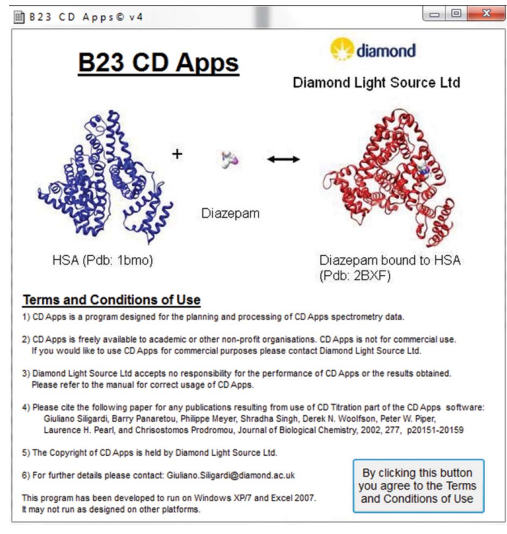

OPEN Ә ACCESS 